\title{
Fahr's disease: a rare diagnosis requiring admission to the emergency department
}

\section{Mahmut Firat Kaynak}

Department of Emergency Medicine, Bursa Yuksek Ihtisas Training and Research Hospital, Bursa, Turkey

\begin{abstract}
Fahr's disease is a rare degenerative neurological disorder characterized by the presence of abnormal calcium deposition and associated cell loss in the areas of the brain that control movement, including the basal ganglia and cerebral cortex. Clinical findings associated with Fahr's disease include parkinsonism, dystonia, chorea, ataxia and psychiatric symptoms. Fahr's disease may result from metabolic disorders, especially parathyroid disorders. We report our experience with 4 patients admitted to our emergency department with complaints such as convulsions, hand spasms, loss of consciousness, and weakness. Computed tomography of all patients showed calcification in the bilateral basal ganglia. The purpose of this paper is to draw attention to a rare disorder involved in the etiology of elderly patients admitted to the emergency department for seizure and/or unconsciousness..
\end{abstract}

Keywords: Fahr's disease; hypocalcemia; intracranial calcification; seizure; unconsciousness

\section{Introduction}

Fahr's disease (FD) is an inherited neurological disorder characterized by calcification of the basal ganglia and other areas of the brain, parkinsonism, and neuropsychiatric symptoms, which was first described in 1930 [1]. Fahr's disease may result from metabolic disorders, especially parathyroid disorders [1]. Fahr's syndrome (FS) is a general term including Fahr's disease as well as other conditions presenting with secondary calcification of the basal ganglia.

Frequent clinical findings in patients with FD including parkinsonism, dystonia, chorea, ataxia, dementia, and mood disorders [1]. Intracerebral calcifications are usually accompanied by disorders in calcium and phosphorus metabolism. More rarely, FD may occur during the course of other metabolic disorders, familial diseases, or in the absence of abnormalities in calcium metabolism [2-4]. Neuropsychiatric, extrapyramidal and cerebellar symptoms, as well as speech disorders and dementia, may also occur. Some patients are asymptomatic despite the widespread accumulation of calcium. Typically, age at onset of clinical symptoms is $40-60$ years, although these symptoms have also been observed in children [1-4]. 
The aim of this paper is to report our experience with four patients admitted to our emergency department for complaints such as convulsions, hand spasms, loss of consciousness, and weakness, who were diagnosed with FD.

\section{The Presentation of the Patients}

\section{Patient 1}

An 82-year-old woman was transported via ambulance to the emergency department with convulsions and hand spasms. She was reported to have experienced a convulsion two months earlier. Her vital signs were stable at admission. Family members reported that, during the previous few years, she demonstrated remarkable impairments in memory functions and daily activities, including difficulties incommunication and self-care. Serum analysis showed a calcium concentration of $6.3 \mathrm{mg} / \mathrm{dl}$ (normal: 8.4-10.2 $\mathrm{mg} / \mathrm{dl}$ ), phosphorus concentration of 5.3 $\mathrm{mg} / \mathrm{dl}$ (normal: $2.3-4.7 \mathrm{mg} / \mathrm{dl}$ ), and a parathyroid (PTH) concentration of $1.5 \mathrm{pg} / \mathrm{ml}$ (normal: 15-65 $\mathrm{pg} / \mathrm{ml}$ ). Computed tomography (CT) scans showed symmetrical calcifications in the bilateral cerebellar hemispheres at the bulbar and periventricular levels, in the bilateral internal capsule and caudate nucleus, and on both sides at the level of the centrum semiovale (Figure 1). The patient was diagnosed with FD associated with idiopathic hypoparathyroidism. After calcium replacement therapy, the patient was discharged from the hospital for outpatient follow-up.

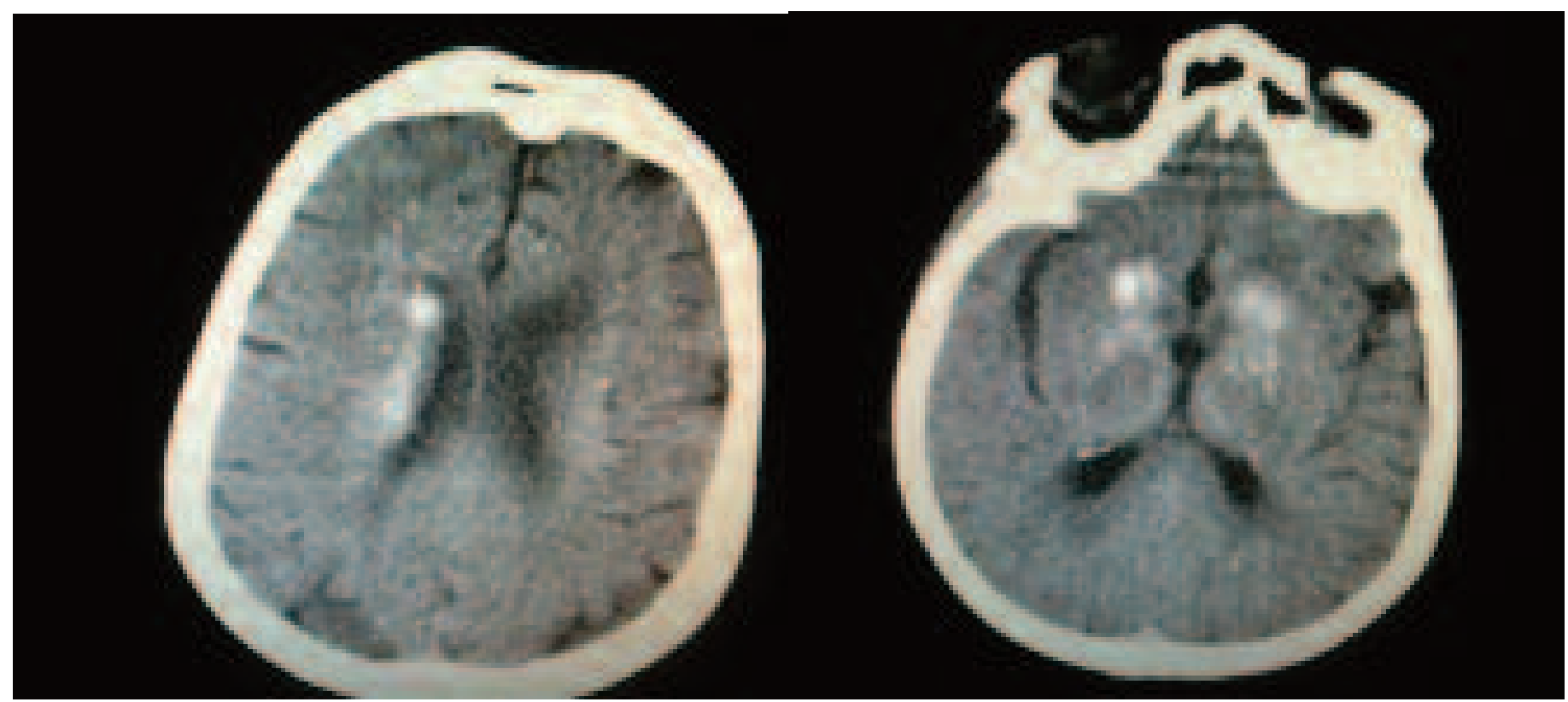

Figure 1. CT scans of patient 1, showing symmetrical calcifications on the bilateral cerebellar hemispheres, at the bulbar and periventricular levels, the bilateral internal capsule and caudate nucleus and on both sides at the level of the centrum semiovale.

\section{Patient 2}

A 40-year-old male was admitted to our hospital for general body fatigue and muscle cramps and numbness in the arms. Upon physical examination, the patient was conscious, alert, oriented and had stable vital signs. His medical history showed no evidence of any comorbid diseases, drug use or allergy. He had been occasionally referred to a doctor for these symptoms and found to have low calcium levels, for which he received oral calcium therapy, but he was not followed-up. At admission, this patient showed evidence of neuromuscular hyperexcitability, with positive Chvostek and Trousseau signs, with blood tests showing serum calcium, phosphorus and PTH

concentrations of $4.08 \mathrm{mg} / \mathrm{dl}$ (normal: $8.4-10.2 \mathrm{mg} / \mathrm{dl}$ ), $5.4 \mathrm{mg} / \mathrm{dl}$ (normal: $2.3-4.7 \mathrm{mg} / \mathrm{dl}$ ) and $6.5 \mathrm{pg} / \mathrm{ml}$ (normal: $15-65 \mathrm{pg} / \mathrm{ml}$ ), respectively. CT showed hyperdense patchy calcifications at the posterior fossa level, the dentate nucleus of both cerebellar hemispheres, the basal ganglia, the thalamus, and at the levels of the frontal, parietal and occipital lobes of the deep subcortical area extending to the white matter (Figure 2). The patient was diagnosed with FD associated with idiopathic hypoparathyroidism. Calcium replacement therapy increased his serum calcium levels, resulting in the resolution of his symptoms. 

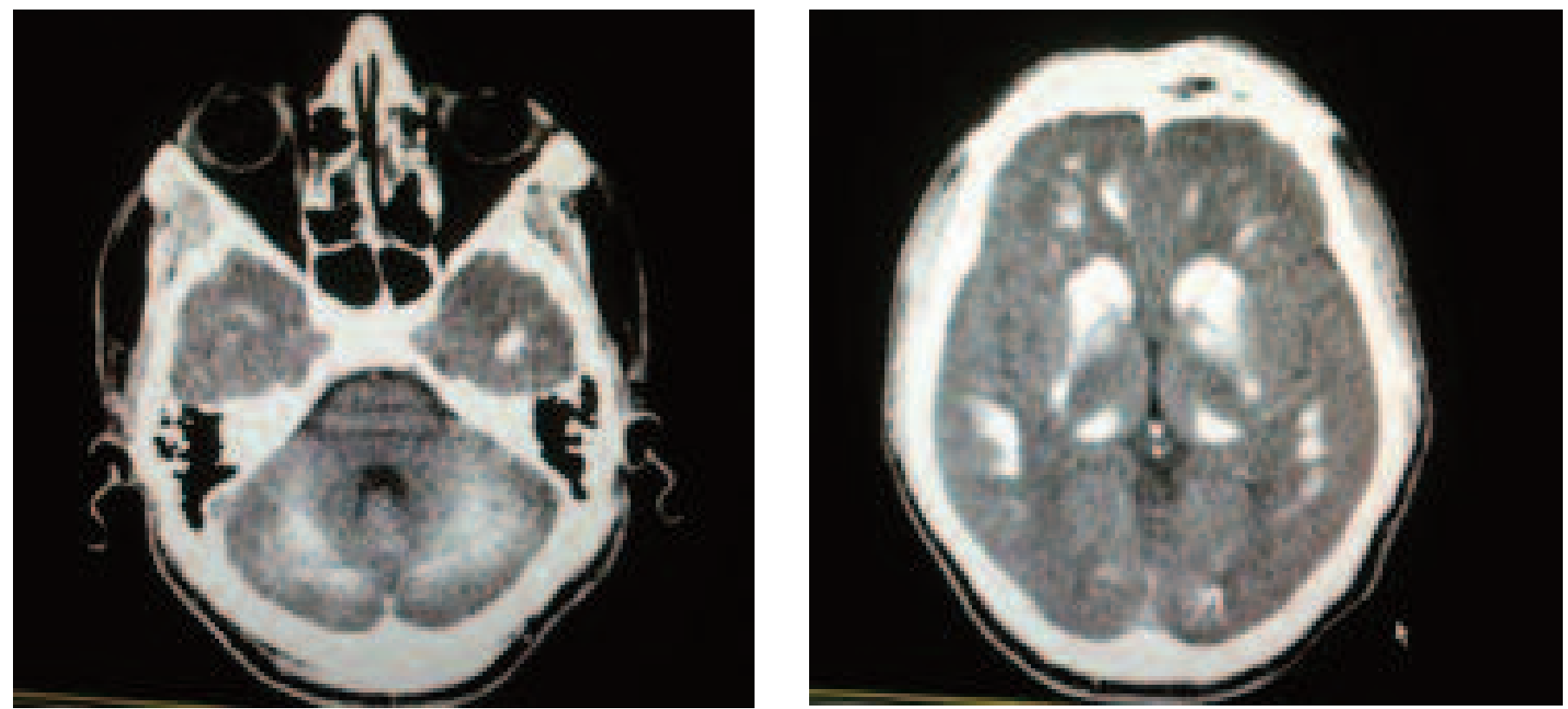

Figure 2. CT scans of patient 2, showing hyperdense patchy calcifications at the posterior fossa level, the dentate nucleus of both cerebellar hemispheres, the basal ganglia, the thalamus, and at the levels of the frontal parietal and occipital lobes of the deep subcortical area extending to the white matter.

\section{Patient 3}

A 56-year-old male with seizures was admitted to the emergency department. His medical history showed increasing dementia, but he had not been diagnosed with epilepsy. His family regarded his condition as age-related. His serum calcium, phosphorus, and PTH concentrations were $6.47 \mathrm{mg} / \mathrm{dl}$ (normal: $8.4-10.2 \mathrm{mg} / \mathrm{dl}$ ), $4.5 \mathrm{mg} / \mathrm{dl}$ (normal: $2.3-4.7$ $\mathrm{mg} / \mathrm{dl}$ ) and $17 \mathrm{pg} / \mathrm{ml}$ (normal: 15-65 pg/ml). He was started on intravenous calcium gluconate therapy. CT showed massive calcification of the bilateral basal ganglia, the periventricular white matter of the thalamus, the centrum semiovale, the posterior fossa

and the bilateral cerebellar hemispheres (Figure 3). The patient was diagnosed with FS.

\section{Patient 4}

A 30-year-old male with generalized tonic-clonic seizures was brought to the emergency service by ambulance. This patient had no previous history of epilepsy, trauma, drug use, alcohol abuse, smoking or previous surgery. Physical examination revealed no pathology. His serum calcium, phosphorus, and PTH concentrations were $9.3 \mathrm{mg} / \mathrm{dl}$ (normal: $8-10.2 \mathrm{mg} / \mathrm{dl}$ ), $4.1 \mathrm{mg} / \mathrm{dl}$ (normal: 2.3-4.7 mg/dl) and $22 \mathrm{pg} / \mathrm{ml}$
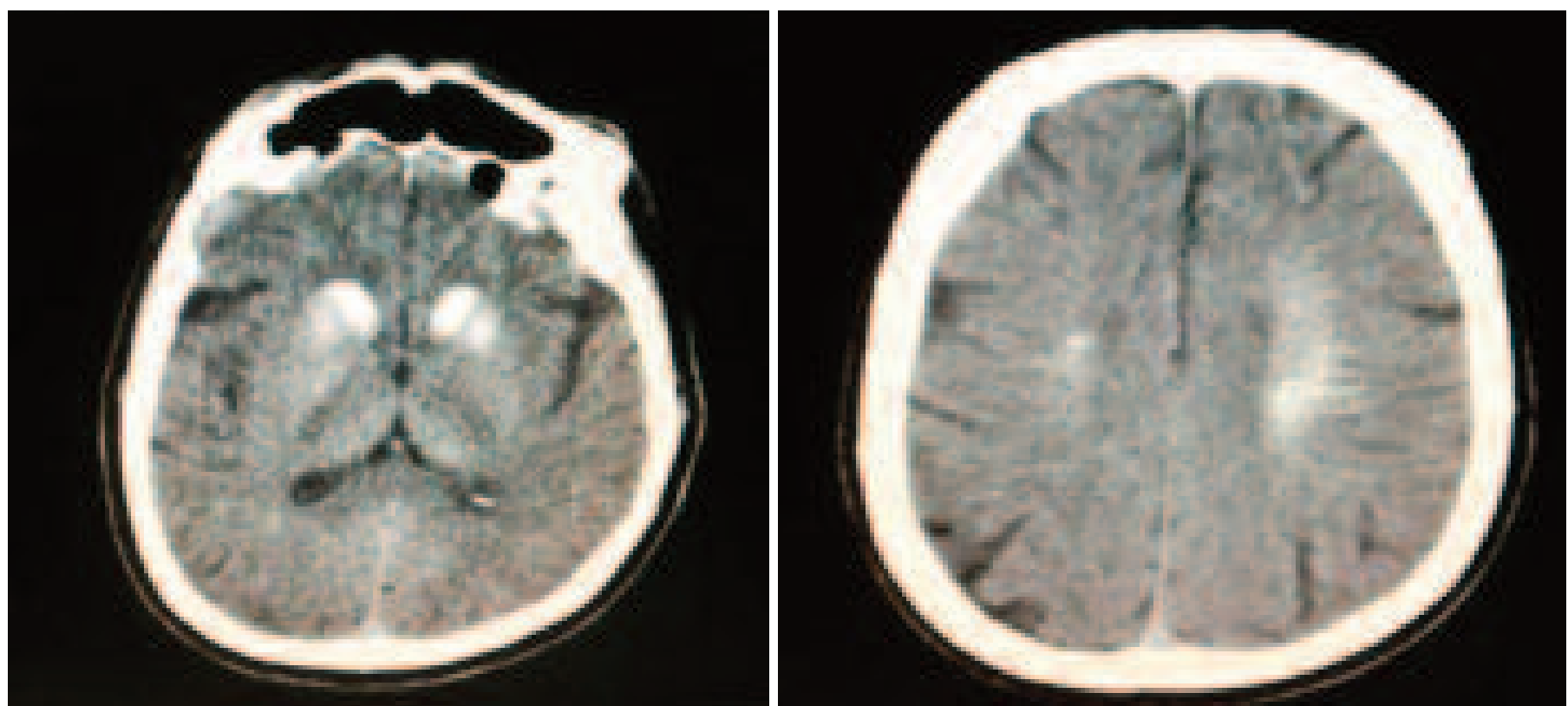

Figure 3. Brain CT scans of patient 3, showing massive calcifications of the bilateral basal ganglia, the periventricular white matter of the thalamus, the centrum semiovale, the posterior fossa and the bilateral cerebellar hemispheres. 


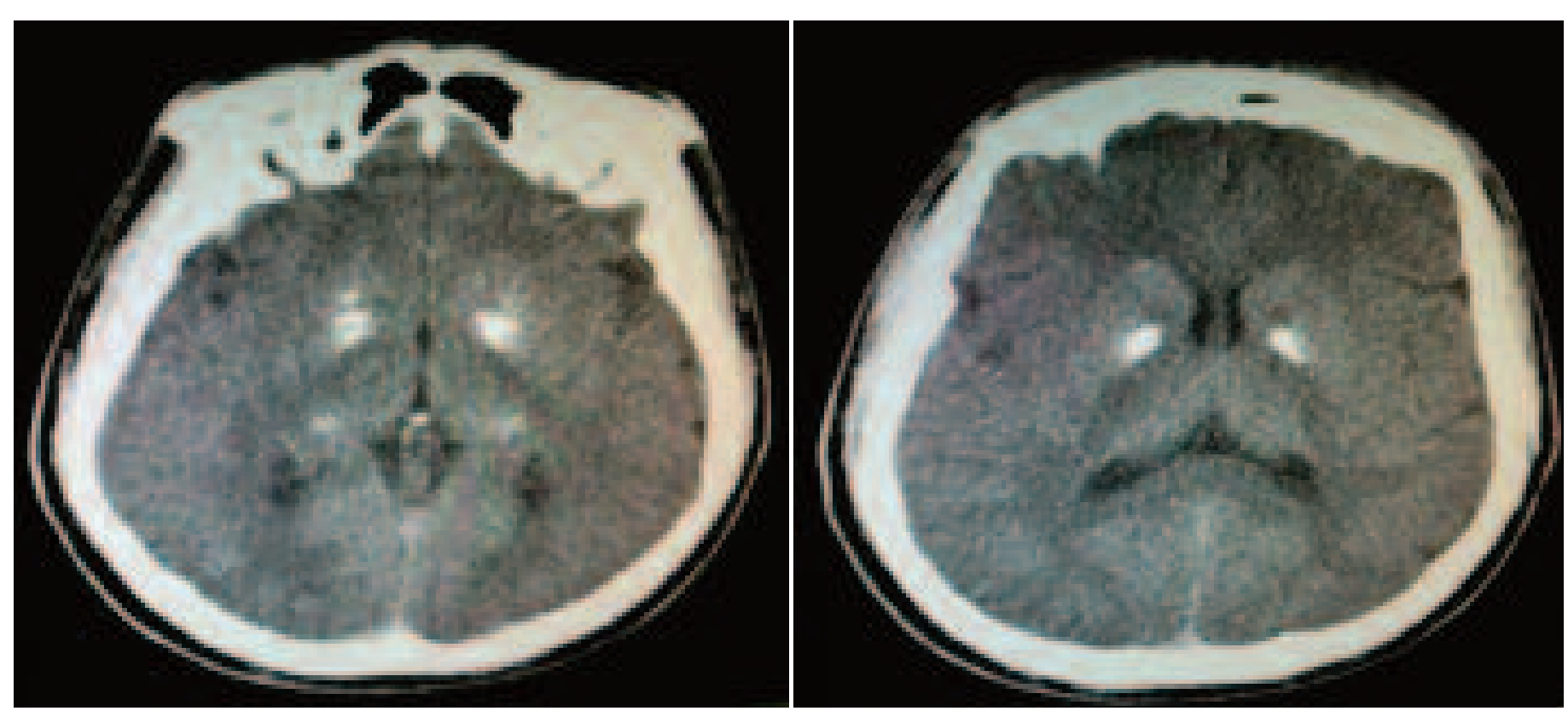

Figure 4. CT scans of patient 4, showing calcification of the bilateral basal ganglia and caudate nucleus.

(normal: 15-65 pg/ml), respectively. His hemogram, serum electrolytes, liver function tests and renal function tests were all within normal limits. Serological tests showed that he was negative for hydatid, toxoplasma, cysticercosis, cryptococcosis, cytomegalovirus and human immunodeficiency virus (HIV). CT of the brain revealed extensive calcification involving the bilateral basal ganglia and caudate nucleus (Figure 4). There was no family history of a similar illness. The patient was diagnosed with FS. Due to his young age and for his clinical well-being, the patient was discharged and followed up as an outpatient.

\section{Discussion}

FS is a condition characterized by symmetrical calcifications of the basal ganglia and cerebellar regions [1]. FD is a type of FS. Although the etiological factors of FS are not clearly understood, it has been associated with disorders of calcium metabolism, degenerative congenital developmental abnormalities and genetic disorders, systemic inflammatory diseases, and toxic effects on anoxic brain interactions. Clinical diagnosis of this condition is based on clinical features, the results of brain imaging, and the exclusion of other causes of intracranial calcification. The most common method used to diagnose FS is CT $[5,6]$. Imaging results showing symmetric and extensive calcification are usually observed, as in our four patients. Two of these patients were diagnosed with FS and two with FD, depending on the intensity and location of the calcifications.

FD/FS can develop secondary to toxic and anoxic effects, congenital degenerative development anomalies, systemic diseases and previous inflammatory events [7-9]. Although most frequently associated with hypoparathyroidism and hypocalcemia [3], the method by which hypoparathyroidism leads to intracranial calcification has not been determined [6]. Initially asymptomatic patients may become symptomatic over time [10].

Manyam et al. [11] reported movement disorders in $56 \%$ and seizures in $22 \%$ of cases in a review of 213 patients with this disorder. Three of our patients were admitted to the hospital with seizures, with a calcium metabolism disorder diagnosed after detailed examination. Findings in two of three patients show that hypoparathyroidism can result in generalized seizures. The incidence of basal ganglia calcification increases as the duration of hypocalcemia increases, with symptoms of calcification emerging after about 30 years [11]. Replacement of calcium and vitamin D improves metabolic abnormalities and delays clinical progression $[12,13]$.

Forty percent of patients with basal ganglia calcifications presents with psychiatric symptoms at the beginning of the disease. Among these; cognitive and psychotic disorders are most prominent [14-16]. Our first patient during the previous few years demonstrated remarkable impairments in memory functions and daily activities, including difficulties 
incommunication and self-care.

\section{Conclusion}

In conclusion, FS/FD should be considered in the differential diagnosis of patients admitted to the emergency department with neuropsychiatric symptoms, a history of undiagnosed seizures, and symptoms of hypocalcemia.

\section{Informed Consent}

Written informed consent was obtained from the patients for the publication of these case series.

\section{Conflict of interest}

The author declared that there are no potential conflicts of interest with respect to the research, authorship, and/or publication of this article.

\section{References}

[1] Paprocka J, Jamroz E, Wackerman-Ramos A, Sokol M, Marszal E. [Norological Picture and $1 \mathrm{H}$ MRS in 4 children with hypoparathyroidisim]. PrezeglLek 2005;62:680-4. [Article in Polish]

[2] Manyam BV. What is and what is not 'Fahr's disease'. Parkinsonism Relat Disord 2005; 11:73-80.

[3] Kokes U, Hiz F, Ozden DL, Cinar M. Psodohipoparatroidinin yol actigi bir Fahr sendromu olgusu. Parkinson Hastaligi Hareket Bozukluklari Dergisi 2009;12;18-24.

[4] Sambrook MA, Hill LF, Cerebrospinal fluid absorbtion in primary hypoparathroidisim. J Neurol Neurosurg Physchiatry 1997;40:1015-7.

[5] Kazis AD. Contribution of CT scan to the diagnosis of Fahr's syndrome. Acta Neurol Scand 1985;71:206-11.

[6] Windeck R, Menken U. Benker G, Reinwein D.Basal ganglia calcification in pseudo hypoparathyroidism type II. Clin Endocrinol (Oxf.) 1981;15:57-63.

[7] Morgante L, Vita G, Meduri M, Di Rosa AE, Galatioto S, Coraci MA, et al. Fahr's syndrome: local inflammatory factors in the pathogenesis of calcification. J Neurol 1986;233:19-22.

[8] Anderson JR. Intracerebral calcification in a case of systemic lupus erythematosus with neurological manifestations. Neuropathol App Neurobiol 1981;7:161-6.

[9] Illum F. Calcification of basal ganglia following carbon monoxide poisoning. Neurodiology 1980;19:213-4.

[10] Harati Y, Jackson JA, Benjamin E. Adult onset idiopathic familial brain calcifications. Arch Intern Med 1984;144:2425-7.

[11] Manyam BV, Walters AS, Narla KR. Bilateral striopallido dentate calcinosis: clinical characteristics of patients seen in a registry. Mov Disord 2001;16:258-64.

[12] Ramonet D, de Yebra L, Fredriksson K, Bernal F, Ribalta T, Mahy N. Similar calcification process in acute and chronic human brain pathologies. J Neurosci Res 2006;83:147-56.

[13] Karimi M, Habibzadeh F, De Sanctis V. Hypoparathyroidism with extensive intracerebral calcification in patients with beta-thalassemia major. J Pediatr Endocrinol Metab 2003;16:883-6.

[14] Harrington MG, Macpherson P, Mcintosh WB, Allam BF, Bone I. The significance of the incidental finding of basal ganglia calcification on computed tomography. J Neurol Neurosurg Psychiatry 1981;44:116870.

[15] Taxer F, Haller R, Konig P. Clinical early symptoms and CT findings in Fahr syndrome. Nervenarzt 1986;57:583-8.

[16] Ogi S, Fukumitsu N, Tsuchida D, Uchiyama M, Mori Y, Matsui K. Imaging of bilateral striopallidodentate calcinosis. Clin Nucl Med 2002;27:721-4. 